\title{
Non Operative Management of Isolated Blunt Liver Trauma: A Task of High Skilled Surgeons
}

\author{
Abdallah Mohamed Taha ${ }^{1, *}$, Ahmed Mohamed Abdallah², Mostafa Mohamoud Sayed ${ }^{2}$, \\ Salah Ibrahim Mohamed ${ }^{2}$, Mostafa Hamad ${ }^{2}$ \\ ${ }^{1}$ General Surgery Department, Faculty of Medicine, South Valley University, Qena, Egypt \\ ${ }^{2}$ General Surgery Department, Faculty of Medicine, Assuit University, Assiut, Egypt
}

Email address:

abdallahsurgery@yahoo.com (A. M. Taha)

${ }^{*}$ Corresponding author

To cite this article:

Abdallah Mohamed Taha, Ahmed Mohamed Abdallah, Mostafa Mohamoud Sayed, Salah Ibrahim Mohamed, Mostafa Hamad. Non Operative Management of Isolated Blunt Liver Trauma: A Task of High Skilled Surgeons. Journal of Surgery.

Vol. 5, No. 6, 2017, pp. 118-123. doi: 10.11648/j.js.20170506.16

Received: September 30, 2017; Accepted: October 16, 2017; Published: December 8, 2017

\begin{abstract}
Background: Liver is the most injured organ in abdominal trauma. The management of blunt liver trauma has markedly changed in the last three decades with a significant improvement in outcomes, due to improvements in diagnostic and therapeutic aids. This study details incidence, grades, causes, types and management of blunt isolated liver trauma in trauma patients admitted to Assiut and South Valley University Hospitals. Patients and Methods: All patients having blunt liver trauma were admitted, history taking, laboratory investigations and resuscitation were performed simultaneously along with ultrasound and CT scan as needed. Data of mechanism trauma, grade of liver injury, method of intervention (Operative or non-operative) and outcome were collected, tabulated and analyzed. Results: Total 174 cases were included in this study with diagnosis of isolated blunt hepatic injuries, mostly young patients were involved, and their mean age was found 24.19+14.65 years. The majority of patients were males $138(79.31 \%)$. Operative management was adopted in 39 patients $(22.41 \%)$, nonoperative management adopted in 129 patients (74.13\%), and 6 patients (3.45\%) died during initial resuscitation. Most cases of liver trauma were found to be grade-III hepatic injury (41.1\%). Chest infection was the commonest complication after surgical management. The mortality rate $(12.1 \%)$ was significantly associated with severity of injury (grade IV and V). Conclusion: Non-operative management of isolated blunt liver trauma is feasible and safe in haemodynaically stable patient with grade I-III liver injury. Mortality in grades IV and V liver trauma is significantly high, so, early operative intervention is recommended in those patients.
\end{abstract}

Keywords: Blunt Liver Trauma, Non-operative Management, Operative Management

\section{Introduction}

The liver is the most frequently injured abdominal organ in blunt trauma. Incidence of liver trauma associated with other solid organ, bowel, mesenteric and diaphragmatic injury has been reported to be $15-20 \%$ [1].

The right lobe is more involved site is the, posteriorsuperior segments particularly, because it is the more voluminous portion of the liver; posterior superior hepatic segments are proximal to fixed anatomical structures such as ribs and spine that may have an important role in producing the lesion [2].
Initial resuscitation must be done for all patients with blunt liver injuries, then, decision is taken whether to manage the patient surgically or not. Non operative management (NOM) in blunt liver trauma is recommended in haemodynamically stable patients with absence of other injuries requiring surgery and absence of peritonitis. Serial physical examination and laboratory testing are considered the pillars in evaluating patients undergone to NOM [3]. Although most hepatic injuries can be safely treated by non-operative methods, many patients become haemodynamically unstable, 
even with a low grade hepatic trauma, necessitating urgent laparotomy [4].

A CT scan of the abdomen with intravenous contrast is the best diagnostic tool for hemodynamically stable patients for both diagnosis and management of blunt hepatic trauma [5]. Hemodynamically stable patients with free intraperitoneal extravasation should be considered for immediate angiography if readily available. [6]

Urgent operative management should be considered in all patients with unstable vital signs or there is the possibility of other injuries requiring surgery. In presence of life threatening major haemorrhage more aggressive procedures can be adopted. These include hepatic manual compression and hepatic packing, ligation of vessels in the wound, hepatic debridement, balloon tamponade, shunting procedures, or hepatic vascular isolation. It is a must to maintain intraoperative intensive resuscitation aiming to prevent or reverse the lethal triad of acidosis, hypothermia and coagulopathy [7].

Selective non-operative management of blunt hepatic injury is associated with lower hospital cost, earlier discharge, avoiding non-therapeutic celiotomies and their associated cost and morbidity, fewer intra-abdominal complications, and reduced transfusion rates $[8-10]$.

\section{Patients and Methods}

\subsection{Aim}

Prospective descriptive study to evaluate the incidence, grades, causes, types and management of blunt liver trauma in patients admitted to Trauma Units in Assiut and South Valley University Hospitals, from September 2016 to September 2017.

\subsection{Selection Criteria for Non-operative Management}

1- Hemodynamically stable patient.

2- Isolated liver injury, no other injuries requiring surgery.

3- Absence of peritonitis.

4- Availability of CT and ICU.

\subsection{Management Plan}

All patients with blunt abdominal trauma managed by history taking, resuscitation and Focused Abdominal Ultrasonography for Trauma (FAST) simultaneously. If free intra-peritoneal fluid was detected in haemodynamically unstable patient, the patient was shifted immediately to the theater for laparotomy. When liver injury was found, different maneuvers were performed to stop bleeding according to the type of injury, as hepatic packing, ligation of vessels in the wound (Figure 1), mattress sutures (Figure 2) or hepatic resection (Figure 3 a \& b). Pringle's maneuver (Occlusion of the hepato-duodenal ligament) was performed in all operated cases to minimize blood loss (Figure 4).

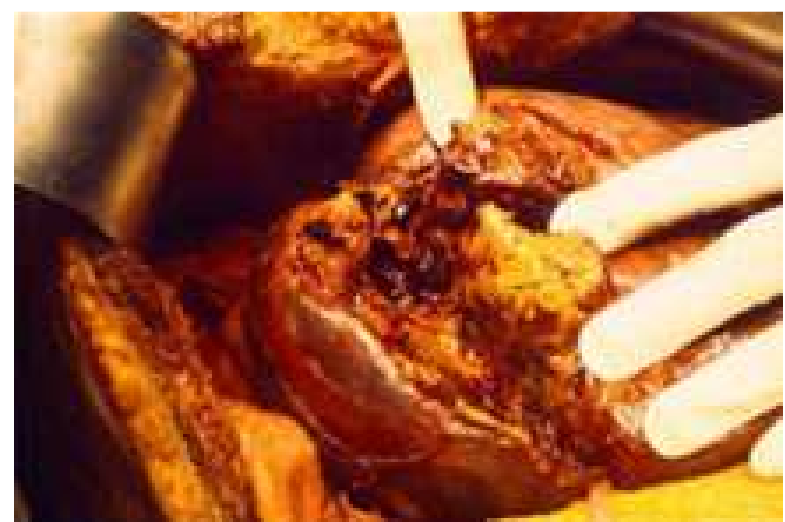

Figure 1. Grade III liver injury in segments V-VIII treated by hepatotomy and vessel ligation.

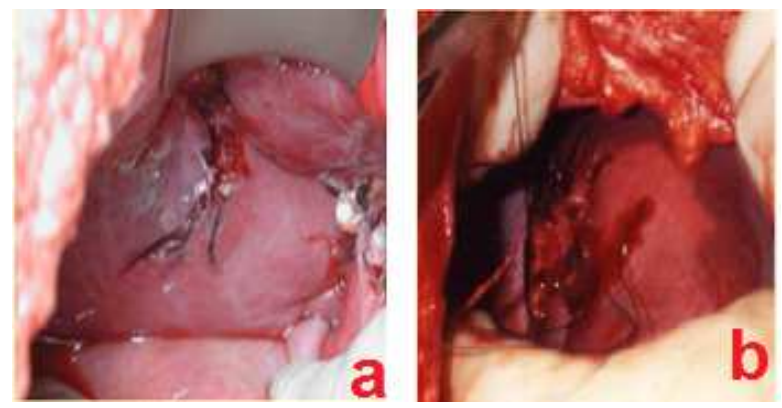

Figure 2. Mattress suture for lacerated lver injury.

a. Stellate liver fracture following blunt trauma.

b. Repair with transverse mattress sutures.

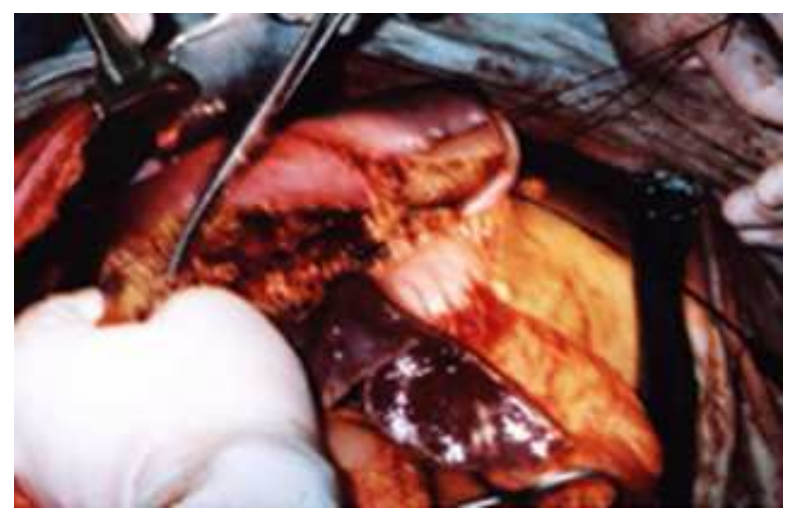

Figure 3a. Partial Resection for grade IV liver trauma.

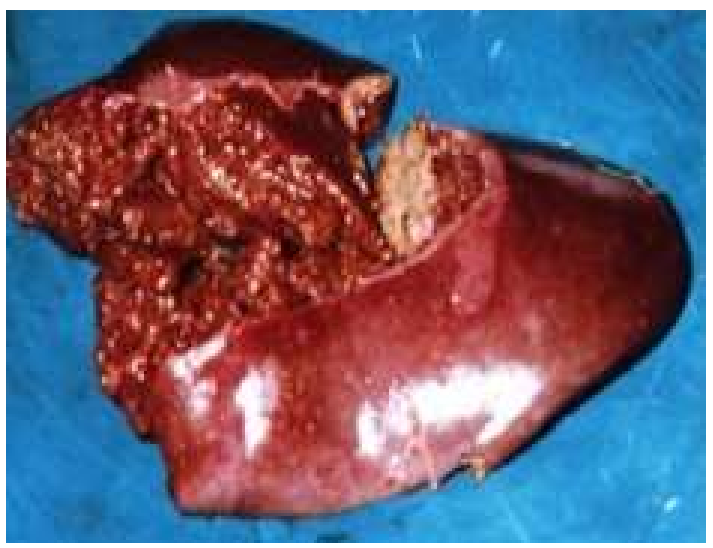

Figure 3b. Specimen for non-anatomical liver resection. 


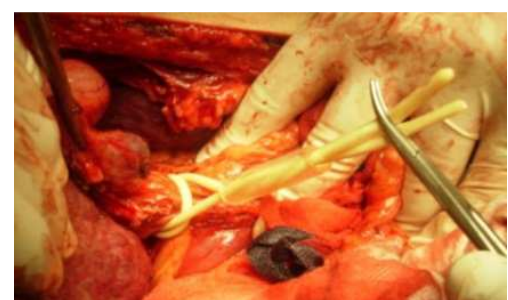

Figure 4. Pringle's maneuver as we performed.
If free intra peritoneal fluid was found in FAST examination in haemodynamically stable patients, abdominal CT with intravenous contrast was done to determine the anatomic liver injury and any other associated injuries.

Liver injuries were graded by a consultant radiologist using American Association for the Surgery of Trauma (AAST) grading scale (Table 1) [11].

Table 1. AAST Liver Trauma Classification [11].

\begin{tabular}{lll}
\hline Grade & Injury type & Injury description \\
\hline I & Haematoma & Subcapsular $<10 \%$ surface \\
& Laceration & Capsular tear $<1 \mathrm{~cm}$ parenchymal depth \\
II & Haematoma & Subcapsular $10-50 \%$ surface area; intraprenchymal, $<10 \mathrm{~cm}$ diameter \\
& Laceration & $1-3 \mathrm{~cm}$ parenchymal depth, $<10 \mathrm{~cm}$ in length \\
III & Haematoma & Subcapsular $>50 \%$ surface area or expanding, ruptured subcapsular or parenchymal haematoma. Intraprenchymal \\
& Laceration & $>3 \mathrm{~cm}$ parenchymal depth \\
IV & Laceration & Parenchymal disruption $25-75 \%$ of hepatic lobe \\
V & Vascular & Juxtavenous hepatic injuries i.e. retrohepatic vena cava/centrl major hepatic veins \\
\hline
\end{tabular}

Patients with systolic blood pressure $>100 \mathrm{mmHg}$ and heart rate less than $90 \mathrm{BPM}$, on three successive measures, either on admission or after 2 liters crystalloid infusion, was regarded as hemodynamically stable.

All patients were admitted in the intensive care unit (ICU) for close observation and rest in bed strictly for at least 3 days. Serial hematocrit and hemoglobin percentage every 6 hours and abdominal US was done daily. When hematocrit and haemoglobin concentration was stable and the follow-up abdominal US not worsened, the patients were shifted to the ward.

Patients discharged after 1 week if no significant changes in hematocrit and haemoglobin concentration and US findings, with clear instructions to restrict physical activities at home for one month from the time of injury.

Non operative management was discontinued if the patient became haemodynamically unstable, had a significant fall in hematocrit and hemoglobin concentration, or intra-abdominal hollow viscus injury was suspected.

\subsection{Outpatient Follow-up}

Weekly visit in the outpatient clinic to perform hematocrit and haemoglobin concentration and abdominal US till no collection.

Data on mechanism of trauma, grade of injury, interventions and outcomes (complications and mortality) were collected, tabulated and analyzed.

\subsection{Statistical Analysis}

Statistical package SPSS version 20 (SPSS, Chicago, IL, USA) was used to analyze data. Data was expressed as numbers, percentages, and arithmetic mean (M) and standard deviation (SD). Chi Square was used to determine significance for categorical variable. $P$ value $<0.05$ was considered significant.

\section{Results}

Total of 174 cases were included in this study with diagnosis of isolated blunt hepatic injury, mostly young patients were involved, and their mean age was $24.19+14.65$ years. The majority of patients were males 138 (79.31\%), while female were $36(20.68 \%)$.

Total of 55080 patients were presented to trauma unit of Assiut and South Valley University Hospitals, 35013 patients were admitted, of these there were 174 patients with liver injury confirmed by US, CT and /or surgical exploration.

The incidence of liver trauma was $0.31 \%$ of all trauma unit presentation, and $0.49 \%$ of all admitted trauma cases. Road traffic accidents was found to be the most common cause of trauma $(59.8 \%)$, the second common cause was falling from height (Table 2).

Table 2. Mechanism of trauma in study group.

\begin{tabular}{lcc}
\hline Mechanism of trauma & Number of patients (174) & Percentage \\
\hline Road traffic accident & 104 & $59.8 \%$ \\
Fall from Height & 50 & $28.8 \%$ \\
Run over by moving vehicle & 9 & $5.2 \%$ \\
Bicycle accident & 7 & $4 \%$ \\
Direct blow to the abdomen & 4 & $2.2 \%$ \\
\hline
\end{tabular}

On initial clinical examination, most patients $(39.65 \%)$ had Localized tenderness to the right hypochondrium and epigastrium $(69.65 \%)$, the second common presentation was shock on admission in $25.85 \%$ of patients (Table 3 ).

Table 3. Initial Clinical assessment on admission.

\begin{tabular}{lc}
\hline Clinical assessment & No. of patients (174) \\
\hline Normal clinical examination & $15(8.62 \%)$ \\
Shock on admission & $45(25.86 \%)$ \\
Localized tenderness to the right & $69(39.65 \%)$ \\
hypochondrium and epigastrium & $45(25.86 \%)$ \\
Generalized abdominal rigidity & \\
\hline
\end{tabular}


US examination to the abdomen was performed in all cases. Its accuracy was $66.52 \%$ to detect the present liver injury. Although, it was highly sensitive in detecting hemoperitoneum $(100 \%)$.

CT demonstrated variable grades of liver injuries. In operated cases, comparing the pre-operative CT with the intra-operative findings, liver injury was found to be accurately graded in 21 patients $(53.84 \%)$, overestimated it (mostly by one grade) in 9 patients $(23.08 \%)$ and underestimated the grade (mostly by one grade) in 9 patients (23.08\%).

Operative management was adopted in 39 patients (22.41\%), 129 patients $(74.13 \%)$ were managed nonoperatively and 6 patients $(3.45 \%)$ died during initial resuscitation (Table 4).
Table 4. Surgical procedures in operative group.

\begin{tabular}{lcc}
\hline Surgical procedure & $\begin{array}{c}\text { Number of } \\
\text { patients (39) }\end{array}$ & Percentage \\
\hline Simple hemostatic sutures + Gel Foam & 15 & $38.46 \%$ \\
Peri-hepatic packing & 9 & $23.1 \%$ \\
Omental patch & 3 & $7.69 \%$ \\
Resectional debridement & 6 & $15.38 \%$ \\
Right hepatectomy & 3 & $7.69 \%$ \\
Non bleeding liver injuries & 3 & $7.69 \%$ \\
\hline
\end{tabular}

Patient's outcome: The mortality rate in this study was $12.1 \%$ (21 patients), 6 patients $(3.45 \%)$ died initial during initial resuscitation, 15 patients $(8.6 \%)$ died from causes directly related to the liver injury as uncontrolled bleeding with hypovolemic shock or post-operative disseminated intra-vascular coagulation. The mortality rate was significantly associated with severity of injury (Table 5).

Table 5. Grades of liver injury in study cases and patient's mortality.

\begin{tabular}{|c|c|c|c|c|c|c|}
\hline \multirow{2}{*}{ Grade of liver injury } & \multicolumn{2}{|c|}{ Survived patients $(n=153)$} & \multicolumn{2}{|c|}{ Died patients $(n=15)$} & \multirow{2}{*}{ Total number of patients $(n=168)$} & \multirow{2}{*}{$p$ value } \\
\hline & No. & $\%$ & No. & $\%$ & & \\
\hline Grade I & 24 & 15.86 & 0 & 0 & 24 & \\
\hline Grade III & 66 & 43.13 & 3 & 20 & 69 & 0.001 \\
\hline Grade IV & 21 & 13.72 & 9 & 60 & 30 & \\
\hline Grade V & 0 & 0 & 3 & 20 & 3 & \\
\hline
\end{tabular}

Failure of non-operative management occurred in 3 patients $(2.32 \%)$ due to haemodynamic instability that necessitated urgent surgical intervention. Other complications shown in table 6, resolved spontaneously within a week. Over all hospital stay was about $12.9+3.5$ days, it was comparable between both groups.

Table 6. Complications in the non-operative group.

\begin{tabular}{lll}
\hline Type of complication & Number of patients $(\mathbf{n}=\mathbf{1 2 9})$ & Percentage \\
\hline Failure of the non-operative & 3 & $2.32 \%$ \\
management & 6 & $4.65 \%$ \\
Intra-hepatic biloma & 9 & $6.97 \%$ \\
Hyperbilirubinemia & 18 & $13.95 \%$ \\
Peri-hepatic collection & & \\
\hline
\end{tabular}

Regarding operative group, mortality was $38.46 \%$ due to causes directly related to liver trauma. peri-hepatic collection and hyperbilirubinemia resolved spontaneously within a week (Table 7).

Table 7. Complications in the operative group.

\begin{tabular}{lcc}
\hline Type of complication & Number of patients $(\mathbf{n}=\mathbf{3 9})$ & Percentage \\
\hline Mortality & 15 & $38.46 \%$ \\
Chest infection & 8 & $20.51 \%$ \\
Peri-hepatic collection & 4 & $10.25 \%$ \\
Wound infection & 2 & $5.12 \%$ \\
Hyperbilirubinaemia & 1 & $2.56 \%$ \\
\hline
\end{tabular}

\section{Discussion}

The patients' mean age was $24.19+14.65$ years in this study and was close to that stated by others in different studies. The high incidence of liver trauma in this young age group can be attributed to the high activity and lack of wisdom and experience present in this age group [12].

In this study, US examination was accurate in detecting $65.52 \%$ of liver injuries although it was $100 \%$ sensitive in detecting hemoperitoneum. Nasim A \& Jerome $\mathrm{J}$, found that abdominal US yielded the sensitivity and specificity of this examination are $63-100 \%$ and $95-100 \%$, respectively and accuracy of $97 \%$ in detecting intra-abdominal injury in 1000 patients [13].

CT accurately estimated the grade of liver injury in $53.84 \%$, overestimated it in $23.08 \%$ and underestimated it in $23.08 \%$ of patients undergone operative management in this study. Taourel et al., assigned at operation a liver injury scale to each case and this was correlated to the pre-operative CT findings. They found accurate estimation in 6 patients $(16 \%)$ out of 37 blunt hepatic trauma, overestimation in $51 \%$ and underestimation in 33\% [14].

In this study, 3 patients $(2.32 \%)$ out of 129 patients of nonoperative management group, eventually became haemodynamically unstable and subjected to urgent laparotomy. Demetriades et al., reported on 46 laparotomies performed in a study including 126 blunt hepatic trauma cases, and found that the main indication for surgery was hemodynamic instability (47.8\%). Other indications included peritoneal signs in $28.3 \%$, associated abdominal injuries in $10 \%$ and massive hemoperitoneum in $4.3 \%$ [15].

We found grade III liver injury was the commonest in this series $(41.1 \%)$. Schweizer et al., found that grade IV was the commonest grade of liver injury in their study including 175 patients, this was followed by grade I in $26 \%$ and grade II or III in $22 \%$ of the cases [16]. 
Resectional debridement using the lines of the injury, rather than anatomical planes has been used in 6 patients. The optimum time may be to combine debridement with pack removal, as necrotic tissue will be well demarcated 48 -h after injury [17].

Perihepatic packing was successful in 9 patients along the course of this study. Demetriades et al, reported that perihepatic packing had been established as an acceptable method of managing blunt liver injuries in situations where it is thought that definitive control of hemorrhage cannot be obtained and was currently undertaken in $4-25 \%$ of patients requiring operative management of liver injuries. Packing can also be employed as a damage control strategy in patients who are critically unstable, coagulopathic or acidotic and, therefore would not tolerate prolonged operative procedure Packing has been remarkably effective in controlling major hemorrhage from liver injuries, even in patients with caval or hepatic venous injuries [15].

The morbidity rates reported in this study in operative group coincide with those reported by others. Ayman et al, reported on the occurrence of chest infection in $21.4 \%$ of the cases, perihepatic collection in $15 \%$, and wound infection in $5 \%$ [18]. Yaman et al, reported that chest infection occurred in $29 \%$ of the patients, perihepatic collection in $4 \%$ and hyperbilirubinemia in $1 \%$ [19].

The overall mortality in this study was similar to that reported in other series, higher than the results reported by others and lower than that reported by others. One of the important prognostic factors included the presence of extraabdominal injuries, especially head and chest injuries. This study showed a significant relationship between the grades of liver injury and mortality. Bala M. et al, reported that patients with liver injury grade 5 , those were significantly associated with high rate of mortality p-value 0.001 [20]. In other studies also reported that low grade of injury was associated with low rate of the complications [7].

\section{Conclusion}

Non-operative management of isolated blunt liver trauma is feasible and safe in haemodynaically stable patient with grade I-III liver injury. Mortality in grades IV and $\mathrm{V}$ liver trauma is significantly high, so, early operative intervention is recommended in those patients.

\section{References}

[1] Konstantinos Chatoupis, Glikeria Papadopoulou, and Ioannis Kaskarelis, New technology in the management of liver trauma. Ann Gastroenterol. 2013; 26 (1): 41-44.

[2] Wong YC, Wang LJ, See LC et al., Contrast material extravasation on contrast-enhanced helical computed tomographic scan of blunt abdominal trauma: its significance on the choice, time, and outcome of treatment. J Trauma, 2003; 54: 164-170.

[3] Coccolini FG, Giulia Montori, Fausto Catena, Salomone Di
Saverio, Walter Biffl, Ernest E. Moore, Andrew B. Peitzman, Sandro Rizoli, Gregorio Tugnoli, Massimo Sartelli, Roberto Manfredi and Luca Ansaloni 1 Liver trauma: WSES position paper, Coccolini et al. World Journal of Emergency Surgery. 2015; 10:.39.

[4] Coimbra R, Hoyt DB, Engelhart S et al., Nonoperative management reduces the overall mortality of grades 3 and 4 blunt liver injuries. Int Surg 2006. 91: 251-257.

[5] Fang JF, Wong YC, Lin BC, et al. The CT risk factors for the need of operative treatment in initially stable patients after blunt hepatic trauma. J Trauma. 2006; 61: 547-554.

[6] Hagiwara A, Murata A, Matsuda T, et al. The efficacy and limitations of trans arterial embolization for severe hepatic injury. J Trauma. 2002; 52: 1091-1096.

[7] Kozar RA, Feliciano VD, Moore EE, Moore FA, Cocanour CS, West MA, Davis JW, McIntyre Jr RC. Western trauma association/critical decision in trauma: operative management of blunt hepatic trauma. J Trauma. 2011; 71 (1): 1-5.

[8] Sartorelli KH, Frumiento C, Rogers FB, et al. Non-operative management of hepatic, splenic, and renal injuries in adults with multiple injuries. J Trauma. 2000; 49: 56-61.

[9] Richardson JD, Franklin GA, Lukan JK, et al. Evolution in the management of hepatic trauma: a 25 year perspective. Ann Surg. 2000; 232: 324-330.

[10] Christmas AB, Wilson AK, Manning B, et al. Selective management of blunt hepatic injuries including non-operative management is a safe and effective strategy. Surgery. 2005; 138: 606-611.

[11] Moore EE, Cogbill TH, Jurkovich GJ, Shackford SR, Malangoni MA, Champion HR. Organ injury scaling: spleen and liver (1994 revision). J Trauma. 1995; 38: 323-4.

[12] Thiago Messias Zago; Bruno Monteiro Pereira; Bartolomeu Nascimento; Maria Silveira Carvalho Alves; Thiago Rodrigues Araujo Calderan; Gustavo Pereira Fraga, TCBC-SP Hepatic trauma: a 21-year experience, Rev. Col. Bras. Cir. vol. 40 no. 4 Rio de Janeiro July/Aug. 2013.

[13] Nasim Ahmed and Jerome J Vernick, Management of liver trauma in adults, J Emerg Trauma Shock. 2011 Jan-Mar; 4 (1): 114-119.

[14] Taourel P, Vernhet H, Suau A, Granier C, Lopez FM, Aufort $\mathrm{S}$, et al. Vascular emergencies in liver trauma. Eur J Radiol. 2007; 64: 73-82.

[15] Demetriades D, Hadjizacharia P, Constantinou C, Brown C, Inaba $\mathrm{K}$, Rhee $\mathrm{P}$, et al. Selective nonoperative management of penetrating abdominal solid organ injuries. Ann Surg. 2006; 244: $620-8$.

[16] Schweizer W, Tanner S, Baer HU et al. Management of traumatic liver injuries. Br J Surg 2006; 80:86-88.

[17] Duane, T. M., Como, J. J., Bochichio, G. V., et al. (2004). Reevaluating the management and outcomes of severe blunt liver injury. J Trauma, 57, 494-500.

[18] Ayman ZakiAzzam, Abdel Hamid Gazal, Mohammed I. Kassem, Magdy A. Souror. The role of non-operative management (NOM) in blunt hepatic trauma, Alexandria Journal of Medicine Volume 49, Issue 3, September 2013, Pages 223-227. 
[19] Yaman I, Nazli O, Tugrul T, Isguder AS, Bozdag AD, Bolukbasi H. Surgical treatment of hepatic injury: morbidity and mortality analysis of 109 cases. Hepatogastroenterology 2007; 54 (77):1507-11.
[20] Bala M, Gazalla SA, Faroja M, Bloom AI, Zamir G, Rivkind AI, Almogy G. Complications of high grade liver injuries: management and outcome with focus on bile leaks. Scandinavian journal of trauma, resuscitation and emergency medicine. 2012 Mar 23; 20 (1):20. 